1 Short title: Individuality in seabird migration

2

\title{
Individual consistency in migratory behaviour of a pelagic seabird
}

Takashi Yamamoto ${ }^{1, *}$, , Akinori Takahashi ${ }^{1,2}$, Katsufumi Sato ${ }^{3}$, Nariko Oka ${ }^{4}$, Maki Yamamoto ${ }^{5}$ and Philip N. Trathan ${ }^{6}$

(1)

${ }^{1}$ Department of Polar Science, The Graduate University for Advanced Studies, 10-3

Midoricho, Tachikawa, Tokyo 190-8518, Japan

${ }^{2}$ National Institute of Polar Research, 10-3 Midoricho, Tachikawa, Tokyo 190-8518,

11

Japan

${ }^{3}$ International Coastal Research Center, Atmosphere and Ocean Research Institute, The University of Tokyo, 5-1-5 Kashiwanoha, Kashiwa, Chiba 277-0882, Japan

${ }^{4}$ Division of Natural History, Yamashina Institute for Ornithology, 115 Konoyama, Abiko, Chiba 270-1145, Japan

${ }^{5}$ Department of Bioengineering, Nagaoka University of Technology, 1603-1

Kamitomioka, Nagaoka, Niigata 940-2188, Japan

${ }^{6}$ British Antarctic Survey, Natural Environment Research Council, High Cross, Madingley Road, Cambridge, CB3 0ET, UK

\footnotetext{
†Present address: Arctic Environment Research Center, National Institute of Polar
} Research, 10-3 Midoricho, Tachikawa, Tokyo 190-8518, Japan.

*Corresponding author's e-mail address: taka.y@nipr.ac.jp 


\section{Abstract}

24 Many animals migrate between breeding and wintering areas; however, whether each

25 animal behaves consistently in space and time between consecutive years is less well 26 understood. Furthermore, previous breeding state (successful or failed) is often not 27 considered when attempting to understand consistent individual differences in 28 behaviour that are likely to impact upon the subsequent behaviour. Between 2006 and 292010 , we used geolocators to track the migratory movements of a pelagic seabird, the 30 streaked shearwater Calonectris leucomelas, with individuals $(N=46)$ being followed

31 for two years or more, including 23 birds that had chicks in two seasons and 23 birds in 32 just one season. All individuals, except for one bird, migrated to the same broad 33 wintering areas, and their migratory route as well as the centre of wintering distribution 34 did not change in relation to the previous breeding outcomes. Migration schedules 35 (dates of departure from the breeding colony, southward and northward migrations, and

36 first return to the colony) did not differ significantly between years for individuals that 37 had chicks during both years, while failed individuals left the breeding colony and individuals, including both males and females, over successive years regardless of the

41 previous breeding outcome, and also the timing of first return back to the colony for 42 females that had chicks in the both previous years and eggs in the both following season.

43 This may imply the existence of individual-specific broad time schedules, possibly a 44 circannual rhythm, though ecological conditions might affect the exact timing of the 
actual departure event. Our results present evidence for high levels of individually consistent behaviour for this pelagic seabird outside the breeding season.

Keywords: seabird, migration, individual difference, consistency, geolocator, streaked shearwater, Calonectris leucomelas.

\section{Introduction}

Within the field of animal behaviour, there is a growing interest in identifying individual differences within a population (Bolnick et al., 2003; Austin et al., 2004). Until recently, conspecific individuals have been treated as ecologically equivalent, and population averages have been considered to be sufficient for understanding a species ecological dynamics. However, different individuals may use different resources, even within the same population and species (Bolnick et al., 2003). Therefore, acknowledging individual differences may have potentially profound implications for understanding ecological and evolutionary processes, and indeed conservation objectives and implementation programs (Dingemanse \& Réale, 2005; Schofield et al., 2010).

In contrast to activities observed during the breeding season, individual behavioural differences and its repeatability outside the breeding season are less well documented (reviewed in Bell et al., 2009). Many animals often migrate for more than several hundred kilometers between breeding and wintering areas during the non-breeding period (Le Boeuf et al., 2000; Phillips et al., 2005; Broderick et al., 2007; 
67 Alerstam et al., 2006; Stanley et al., 2012). During this period, individual differences in

68 behaviour (in terms of space and time) within a population and consistency within

69 individuals between consecutive years have been rarely examined due to our inability of 70 following specific animals. This is also true for avian species, even though bird 71 migration has been relatively well studied (Newton, 2008). Spatial and temporal 72 repeatability estimates of migratory birds have been based on observations and 73 recaptures of marked individuals at breeding, wintering, or stopover locations (Møller, 74 2001; Catry et al., 2004; Battley, 2006), and also examined using the stable isotope 75 method (Phillips et al., 2009; Quillfeldt et al., 2010). However, these studies only 76 provide snapshots about repeatability during migration. Also, although satellite-linked 77 tags (PTTs) provide accurate positions over a long time, they can be applied only to 78 relatively large-sized species (e.g. ospreys; Alerstam et al., 2006) due to the size and 79 weight. In addition, recent studies suggest that breeding performance is likely to impact 80 upon the subsequent wintering ecology (and vice versa) (so-called 'carry-over effect'; 81 reviewed by Harrison et al., 2010). However, previous studies often did not consider the 82 breeding outcome for understanding consistent individual differences in behaviour (but 83 see Phillips et al., 2005; Catry et al., 2013).

$84 \quad$ Streaked shearwaters Calonectris leucomelas are a pelagic seabird, that breed 85 in southeast Asia (Oka, 2004). Our previous study examined the migratory behaviour of 86 streaked shearwaters using light-based geolocators, and showed three distinct wintering 87 areas during non-breeding period: the seas off northern New Guinea, Arafura Sea, and 88 South China Sea (Yamamoto et al., 2010). Streaked shearwaters exhibited large 
89 variation in the timing of southward migration between individuals (more than 3 months

90 between the first and last birds) (Yamamoto et al., 2010). Consequently, these results

91 give rise to a question as to whether each individual migrates to the same wintering

92 areas with a fixed migratory schedule between consecutive years. Furthermore, we may

93 expect changes in the subsequent wintering behaviour in relation to prior breeding

94 performance; our previous study examined the migratory behaviour of this species only

95 for one season (Yamamoto et al., 2010). Variation in the wintering destination (3

96 choices) and timing of migration (over 3 months) among a population of streaked

97 shearwaters may provide us with an opportunity to examine repeatability or plasticity in relation to their sex and breeding outcomes.

\section{2. Material and methods}

108 2.1. Bird Tracking

109 Fieldwork was carried out at the breeding colonies on Sangan Island $\left(39^{\circ} 18^{\prime} \mathrm{N}\right.$, $\left.141^{\circ} 58^{\prime} \mathrm{E}\right)$, Mikura Island $\left(33^{\circ} 52^{\prime} \mathrm{N}, 139^{\circ} 14^{\prime} \mathrm{E}\right)$ and Awa Island $\left(38^{\circ} 27^{\prime} \mathrm{N}, 139^{\circ} 13^{\prime} \mathrm{E}\right)$ in 
111 Japan. In streaked shearwaters, arrival at the breeding colony takes place mostly in

112 March, laying occurs from late June to early July, hatching in mid-August, and fledging

113 in mid-November (Oka et al., 2002; Yamamoto et al., 2010, 2012). Only one egg is laid

114 per breeding attempt and there are no replacement clutches. Over a five year period 115 from 2006 to 2010, we hand-captured 100 streaked shearwaters (different individuals)

116 nesting in burrows during the chick-rearing period from late August to late September, 117 and fitted geolocators (Mk4: $25 \times 18 \times 7 \mathrm{~mm}, 4.5 \mathrm{~g}$ or Mk5: $18 \times 18 \times 6.5 \mathrm{~mm}, 3.6 \mathrm{~g}$, 118 developed by the British Antarctic Survey, Cambridge, UK) to the tarsus of each bird 119 using a plastic leg ring (see Figure 1 in Takahashi et al., 2008) (Table 1). All birds were 120 rearing their single chicks at the time of geolocator attachment; individuals with chicks 121 were targeted in this first year to ensure they were breeding birds and not simply 122 prospecting birds that may eventually breed elsewhere. In total, we recaptured 75 123 equipped birds from the respective breeding colonies one year after the attachment 124 during the incubation (from early to mid-August) or chick-rearing (from mid-August to 125 late September). All devices were removed, with new geolocators being attached to 51 126 recaptured birds for a second year of tracking, of which we recaptured and removed 127 devices from 42 birds the following season during the incubation (from early to 128 mid-August) or chick-rearing (from mid-August to late September). For a consecutive 129 year of tracking, we reattached geolocators to the recaptured birds, including some 130 individuals that did not have eggs and/or some failed to hatch eggs. In the second year, 13123 birds (12 males and 11 females) had chicks, but 23 (13 males and 10 females) did not.

132 Note that we recaptured one bird from Sangan Island equipped in 2006 after three years, 
133 and three birds from Mikura Island equipped in 2006 and two birds from Awa Island 134 equipped in 2008 after two years since the first year of geolocator attachment (Table 1). 135 Also, one bird from Sangan Island tracked for the second year with a new geolocator in 1362007 was recaptured during the chick-rearing period after two years since the 137 deployment, obtaining three consecutive year tracking data (please see Table 1 for the 138 details of GLS attachment/recovery numbers). Two loggers (one from Sangan bird and 139 one from Awa bird) had recording errors, and the data were unusable. Consequently, 30 140 birds at Sangan Island ( $N=27$ in 2006-2008, $N=3$ in 2007-2009), four birds at Mikura 141 Island $(N=4$ in 2006-2008), and 10 birds at Awa Island $(N=10$ in 2008-2010) were 142 tracked during two consecutive years, and two birds at Sangan Island were tracked for 143 three consecutive years (2006-2009) (46 birds in total, with 25 males and 21 females).

144 The sex of all the birds was determined based on their vocalizations during handling, as 145 the calls of males are high pitched whereas those of females are low pitched (Arima \& 146 Sugawa, 2004).

147 We monitored chicks of the equipped birds during the chick-rearing period, 148 and determined the breeding outcome (success or failure) of the birds by direct 149 observation of their chicks on the last date of monitoring (usually in the mid-October), 150 as streaked shearwaters raise their chick until early November (Oka et al., 2002). Also, 151 we determined the breeding status (the existence of an egg) of the birds by examining 152 whether the geolocator data included repeated periods of darkness, assumed to represent 153 time spent within the nest burrow during incubation (Yamamoto et al., 2012). 
155 immediately released to their nests after handling. The total mass of the geolocator with 156 the plastic leg ring was $7 \mathrm{~g}(\mathrm{Mk} 4)$ and $6 \mathrm{~g}$ (Mk5), which represents about $1.2 \%$ of the 157 mean body mass of the birds (mean $\pm \mathrm{SD}=576 \pm 73 \mathrm{~g}, N=100$ ) and ca. $1.6 \%$ of the 158 body mass of the smallest bird (435 g). Upon recovery, we removed the logger and 159 plastic leg ring from each individual. We did not observe any injuries to their legs.

\subsection{Data Analysis}

162 Geolocators measure light levels at $60 \mathrm{~s}$ intervals, and record the maximum value 163 during each $10 \mathrm{~min}$ period. Immersion in seawater was measured every $3 \mathrm{~s}$ and 164 compiled over each 10 min period. Water temperature was recorded every 10 min only 165 after continuous immersion for $20 \mathrm{~min}$. Light data were processed following the 166 procedures in Takahashi et al. (2008) and Yamamoto et al. (2010). Day length is used to 167 provide an estimate of latitude, while the relative timing of recorded midday or 168 midnight is used to provide an estimate of longitude (Hill, 1994).

We identified the date of final departure and first return to the breeding colony

170 before and after the migration, respectively, using light and immersion data (Yamamoto 171 et al., 2010). Because streaked shearwaters land at the breeding colony after dusk, and 172 depart the colony before dawn, immersion data were recorded as 0 (out of water) over 173 the substantial period of the night that birds were at the colony. We assumed that birds 174 were migrating south when they crossed a boundary $500 \mathrm{~km}$ south or west of the 175 breeding colony (Yamamoto et al., 2010). Also, we assumed that birds had reached their 
176 final wintering destinations after crossing one of three predetermined boundaries $\left(10^{\circ} \mathrm{N}\right.$

177 for the seas off northern New Guinea, the Equator for the Arafura Sea, and $120^{\circ} \mathrm{E}$ for

178 the South China Sea), as defined in Yamamoto et al. (2010). Similarly, we assumed that

179 birds were migrating north when they flew from one of the three predetermined

180 boundaries to within $500-\mathrm{km}$ of the colony. Immersion data were partially or completely

181 unavailable for two birds, and, thereby, the dates of departure from the breeding colony

182 and/or first return to the colony were not obtained from these birds. In addition, one

183 logger stopped logging light intensity on 26 January 2007; therefore, the location data

184 for this bird were unavailable after that date. At-sea distributions of streaked 185 shearwaters during the post-breeding period were established by fixed kernel density 186 maps using the Spatial Analyst toolkit in ArcGIS 9.3 (ESRI $\left.{ }^{\circledR}\right)$.

187 To inspect the variation in repeatability along the migratory route within 188 individuals, we calculated the mean value of longitude within each $5^{\circ}$ interval of 189 latitude between $30^{\circ} \mathrm{N}$ and $0^{\circ}$ (e.g. for a bird migrated to the seas off northern New 190 Guinea, the mean value of longitude at latitudes of $>25^{\circ}-<30^{\circ},>20^{\circ}-<25^{\circ},>15^{\circ}-<$ $19120^{\circ},>10^{\circ}-<15^{\circ}$, were calculated), and averaged. Then, we calculated the distance 192 between the migratory routes for two years. Similarly, we calculated the distance 193 between the centroids of wintering distribution for two years within the same 194 individuals.

195 Statistical analyses were performed in $\mathrm{R}$ software, version 2.5.1 (R 196 Development Core Team). Because we conducted the study at three different breeding 197 colonies over a five-year period from 2006 to 2010, possible colony and year effects on 
198 the dependent variables (i.e. migratory schedules) were tested by including those as

199 predictors in generalized linear models (GLM) with breeding status as an independent 200 variable. Colony and year never proved to be significant predictors of the dependent 201 variables, and, thus, we did not consider colony and year effects in our analysis. 202 Although annual conditions in the marine environment (e.g. sea surface temperatures) 203 appear to influence the timing of migration for several marine vertebrates (e.g. 204 Sherrill-Mix et al., 2008), breeding outcome and endogenous rhythm probably more 205 significantly influence the broad timing of movement (see Discussion). Also, colony 206 differences, if there is, are probably less apparent due to different sample sizes between 207 the colonies (30 birds at Sangan Island, four birds at Mikura Island, and 10 birds at Awa 208 Island). Effects of the sex and previous breeding outcome on the consistency in 209 migratory route and wintering distribution are examined using GLM. Timings of each 210 migratory event of the same individual between two years were compared using paired 211 t-test. Repeatability in an individual's migratory schedule between years was evaluated 212 using the intraclass correlation coefficient $(r)$ (Zar, 1999). Values are presented as mean $213 \pm \mathrm{SD} ; p$ values $<0.05$ were considered significant.

\section{3. Results}

\section{3.1 Spatial Consistency}

217 Streaked shearwaters migrated to three distinct wintering areas during the post-breeding 218 period: the seas off northern New Guinea, Arafura Sea, and South China Sea (Figure 1). 219 Of the 46 individuals, 45 migrated to the same wintering areas over the two years 
(Figure 2a, c, d, e). Furthermore, two individuals also migrated to the same wintering 221 area for three successive years (Figure 2b). Only one individual (male) changed its 222 wintering destination over two years from the Arafura Sea to seas off northern New 223 Guinea (with $1303 \mathrm{~km}$ separation between the centroids of the two wintering areas) 224 (Figure 2f). In birds exhibiting fidelity to wintering areas, the mean distance between 225 the centroids of the wintering area for two years within the same individuals was $459 \pm$ $226326 \mathrm{~km}$ in males and $259 \pm 204 \mathrm{~km}$ in females. There was no difference in the distance 227 between the centroids of the wintering area for two years depending on the previous 228 breeding outcomes (success or failure), but males showed larger distances compared to 229 females (GLM, breeding outcome: $t=-1.28, p=0.21$, sex: $t=2.47, p<0.05$ ). Individuals followed similar migratory routes between years (Figure 2a-e).

231 The distance between migratory routes for two years within individuals was $299 \pm 138$ $232 \mathrm{~km}$ in males and $252 \pm 177 \mathrm{~km}$ in females for the southward migration as well as $551 \pm$ $233 \quad 341 \mathrm{~km}$ in males and $452 \pm 354 \mathrm{~km}$ in females for the northward migration. There was 234 no difference in the distance between migratory routes for two years in relation to the 235 sex and previous breeding outcome (GLM, all $p>0.30)$. Furthermore, streaked 236 shearwaters from Awa Island appeared to show two different migratory routes when 237 leaving or when approaching Japan (through the Pacific Ocean, $N=8$, vs. through the 238 Sea of Japan, $N=2$; Figure 2c, d, respectively); all individuals took the same route as in 239 the previous year.

\subsection{Temporal Consistency}


242 Migration schedules (dates of departure from the breeding colony, southward and 243 northward migrations, and first return to the colony) did not differ between years for 244 individuals that had chicks during both years (paired t-test, all $p>0.15$ for both males 245 and females). On the other hand, individuals that did not have chicks in the second year 246 left the breeding colony significantly earlier (paired t-test, male: $t_{13}=3.88, p<0.01$, 247 female: $t_{10}=2.69, p<0.05$ ) (Figure 3a), and males started the southward migration at 248 an earlier date $\left(t_{13}=3.07, p<0.05\right)$ than the previous successful year (ca. 12 days 249 earlier) (Figure 3b). Females also started the southward migration slightly earlier (ca. 3 250 days earlier) than the previous year, though it was not significant $\left(t_{10}=0.92, p=0.38\right)$. 251 For these birds, the timing of northward migration and first return to the colony did not 252 significantly differ between previously successful and failed years (northward 253 migration: $t_{13}=-1.47, p=0.17$ for male and $t_{10}=1.12, p=0.29$ for female; first return: $254 t_{13}=-1.18, p=0.26$ for male and $t_{10}=1.03, p=0.33$ for female). Meanwhile, we found 255 a significant between-year individual consistency in the timing of southward migration 256 regardless of the previous breeding outcome (Figure 3b), and also for the first return 257 back to the colony in females that had chicks for the both previous years (Figure 3d). 258 There was no repeatability within individuals in the timing of departure from the colony 259 and northward migration for both groups (Figure 3a, c).

261 subsequent breeding outcome (i.e. whether individuals had eggs in the following 262 season) (paired t-test: all $p>0.13$ for both males and females). Also, there was no 263 repeatability within individuals in the timing of first return for these groups 
264 (repeatability test: all $p>0.07$ ), except for females that had eggs in the both following 265 season $(r=0.59, p<0.05)$.

\section{4. Discussion}

268 The key finding from our study was to reveal the individual consistency in spatial and 269 temporal migratory pattern of a pelagic seabird, and its relation with the breeding 270 outcome before and after the migration, using robust sample sizes $(N=46)$. Recently, 271 using electronic tags, individual consistency in behaviour outside the breeding season, 272 including site fidelity and consistency in the chronology of movement, has been 273 reported for a variety of terrestrial and marine taxa (Bradshaw et al., 2004; Phillips et al., 274 2005; Broderick et al., 2007; Jorgensen et al., 2010; Schofield et al., 2010; Vardanis et 275 al., 2011; Stanley et al., 2012), while some animals also exhibit a level of plasticity 276 (Dias et al., 2011; Guilford et al., 2011). Nonetheless, the number of individuals tracked 277 for more than one year is usually small. Although Bogdanova et al. (2011) showed the 278 differences in winter distribution in relation to the prior breeding outcome, they were 279 unable to present results for the same individuals with a different breeding status 280 (success vs. failure). Only a few studies examined the individual consistency in 281 migratory behaviour in relation to the breeding outcome (Phillips et al., 2005; Catry et 282 al., 2013).

283 Our study provides strong evidence for high levels of individually consistent 284 behaviour for a pelagic seabird migrating within the western Pacific Ocean. Streaked 285 shearwaters showed a high degree of consistency in their wintering area over years. 
286 Furthermore, each individual exhibited fidelity to relatively small-scale areas within the 287 broad wintering areas, and their wintering distributions did not change in relation to the 288 prior breeding outcomes. Recent studies suggest that the fidelity to a specific wintering 289 site is relatively common characteristic among migratory animals (e.g. Bradshaw et al., 290 2004; Phillips et al., 2005; Broderick et al., 2007). However, one out of 46 streaked 291 shearwaters tracked changed the wintering area in the subsequent year in our study, 292 assuming their possible flexibility in migratory behaviour. A previous study suggested 293 that Cory's shearwaters Calonectris diomedea, a closely related species to streaked 294 shearwaters, have several potential wintering areas, and some individuals (36\% of study 295 birds) within a population changed the wintering destination between years (Dias et al., 296 2011). Similarly, streaked shearwaters could change their wintering destination in any 297 given year, but individuals did not or might be unlikely to migrate to different wintering 298 areas over consecutive years. One possibility could be that individuals benefit from 299 multiyear site fidelity to specific foraging areas, as it provides for increased familiarity 300 with local resources (Bradshaw et al., 2004; Newton, 2008). Also, streaked shearwaters 301 wintered in tropical oceans where annual fluctuation of marine environment is usually 302 less pronounced (i.e. predictable) (Longhurst \& Pauly 1998), and, therefore, flexibility 303 would be less advantageous for them compared to species exploits a highly 304 unpredictable environment (Quillfeldt et al. 2010). On the other hand, males showed 305 relatively larger differences in the wintering distribution between two years compared to 306 females. Although the reason of this sex-related difference is unknown, there is a 307 possibility that males are more likely to exploit large areas, as one individual that 
308 changed its wintering destination in our study was also male.

$309 \quad$ Individuals migrated along similar pathways within a few hundred kilometers

310 between the breeding colony and wintering area, and the migratory route did not differ

311 between two years depending on the breeding outcome and sex; though the precision of

312 light-level based geolocation data in estimating location is low ( $\pm 186 \mathrm{~km}$; Phillips et al., 313 2004). This is probably also supported, in part, by the result from individuals from Awa

314 Island that showed two different migratory routes from the breeding colony to the

315 wintering areas (Pacific vs. Japan Sea), and each individual migrated along the same 316 route over two years. The distance between migratory routes for two years was 317 relatively larger during the northward migration, probably because the low accuracy in 318 estimating positions around the spring equinox when most shearwaters were migrating 319 towards the breeding colony from the wintering area. Similarly, route fidelity has been 320 noted in several species (Le Boeuf et al., 2000; Broderick et al., 2007), but also 321 flexibility in route in terrestrial and marine environment (Alerstam et al., 2006; 322 Schofield et al., 2010; Guilford et al., 2011; Vardanis et al., 2011; Stanley et al., 2012). 323 Route fidelity may be affected by conditions en route that are likely to change between 324 years and regions (Bauer et al., 2010), or possibly related to migratory strategy 325 (heritability vs. learning).

Previous studies have reported that failed breeders start their outward 327 migration earlier than do successful breeders (Phillips et al., 2005; Bogdanova et al., 328 2011; Catry et al., 2013), because the timing of departure from the colony is probably 329 constrained by the condition and/or age of their chicks in a given year. Our results 
330 support this; individual streaked shearwaters that did not have chicks in the second year

331 of our study left the breeding colony earlier and appeared to start their southward 332 migration overall earlier than in the previous year when they successfully fledged 333 offspring. Nonetheless, individuals, including males and females, exhibited repeatability 334 in the timing of their southward migration regardless of the previous breeding outcome, 335 with a great variation in the timing between individuals ( 8 October-15 January). This 336 may imply that individual streaked shearwaters have specific time schedules for the 337 timing of their migration, possibly a circannual rhythm (Gwinner, 1996; Newton, 2008). 338 Meanwhile, such an endogenous rhythm could only initiate the preparatory processes, 339 and at a particular date, weather, food, and other ecological conditions might then play 340 an important role in altering the exact timing of the actual departure event (Bradshaw \& 341 Holzapfel, 2007; Newton, 2008), resulting in an overall earlier southward migration in 342 failed birds.

We found no repeatability within individuals for the timing of northward 344 migration. Endogenous rhythms could drift under the conditions of constant daylength 345 in the tropical region (Bradshaw \& Holzapfel, 2007; Newton, 2008) where shearwaters 346 spent the post-breeding period. Birds possibly use other photic cues (e.g. light intensity 347 fluctuation) as found in some tropical birds (Newton, 2008; Goymann et al., 2012), 348 though these are relatively weak compared with the photoperiodic cues (Goymann et al., 349 2012). Also, as repeatability is a measure of individual consistency relative to other 350 individuals in the population, the repeatability was less detectable for the timings of 351 northward migration due to less variability between individuals (13 February-18 
352 March) compared to that for the southward migration, ranged over three months.

353 Females that had chicks in the both previous years exhibited repeatability in the timing 354 of first return back to the breeding colony, while successful males and failed individuals 355 did not. In closely-related Cory's shearwaters, failed birds depart earlier from wintering 356 areas and arrived sooner at the breeding colony, probably because they would be in a 357 more favorable state due to their reduced parental investment during the previous 358 breeding period (Catry et al., 2013). In this study, the timing of northward migration and 359 first return to the colony did not differ between years depending on the previous 360 breeding state (success or failure). Hence, in this respect, the previous breeding state (i.e. 361 parental investment) is less likely to carry over to the subsequent behaviour during the 362 non-breeding period in streaked shearwaters. However, as females that had chicks in the 363 both previous years and that had eggs in the both following season exhibited the 364 repeatability, the breeding state may affect the time schedule within individuals for first 365 return to the colony and vice versa in females, possibly related to their body condition. 366 In males, there was no repeatability in the timing of first return to the colony regardless 367 of the any breeding state. Although the timing of first return to the breeding colony is 368 likely to be an important consequence for breeding attempt, such as nest occupation and 369 reunion of pairs (Kokko, 1999; Gunnarsson et al., 2004), it was not related to the 370 subsequent breeding outcome in males. Streaked shearwaters show a long period of 371 pre-laying (ca. three months), and, during this period, males return to the breeding 372 colony probably to defend their nests from other prospecting birds (Yamamoto et al., 373 2011). Therefore, activities during the pre-laying period may be more critical for 
374 reproductive success in males.

Recent climate-related changes undoubtedly appear to affect behaviour and

376 phenology of a variety of taxa during both breeding and non-breeding period (review by 377 Walther et al., 2002, Austin \& Rehfisch, 2005). Under such situations, plasticity in 378 behaviour would represent an adaptive advantage (Parmesan, 2006, Reed et al., 2010). 379 Individual consistency in behaviour exhibited in streaked shearwaters is possibly 380 disadvantageous for responding to a changing environment. Nonetheless, on the other 381 hand, individual differences in behaviour within a population would allow a certain 382 individuals to cope with such changes (Ghalambor et al., 2007). Also, individual 383 differences in behaviour are considered to occur through time and across situations 384 (Bolnick et al., 2003; Bell et al., 2009), assuming that behaviour during the 385 non-breeding period is also likely to affect, to some extent, behaviour during the 386 breeding period, and vice versa. Examining individual behaviour over the entire annual 387 cycle may therefore provide us with a prime opportunity to understand how individual 388 differences in behaviour shape life history traits and to relate this to fitness.

\section{Acknowledgments}

391 We are grateful to the late Setsuo Kurimoto, to N. Miura, T. Honbo for logistic support 392 in the field. We also thank many colleagues for field assistance. K. Yoda, R. M. Suryan, 393 and two anonymous reviewers provided valuable comments on the manuscript. This 394 work was partially supported by the Japan Society for the Promotion of Science (JSPS) 395 Research Fellowship for Young Scientists (22-1252 to TY), JSPS research grants 
396

(19651100 to AT, 19255001 to KS), Grant-in-Aid for Scientific Research (Special

397 Promotion) of the Ministry of Education, Culture, Sports, Science and 398 Technology-Japan to NO of YIO, Fujiwara Natural History Foundation (2009) to TY 399 (No. 19), and Cooperative Program (No. 110, 2009) of Atmosphere and Ocean Research 400 Institute, The University of Tokyo. The work was conducted with permits from the 401 Ministry of the Environment; No. 060609001, 070718001, 080605001 and 090520001 402 for Sangan Island, 18-340, 19-354 and 20-484 for Mikura Island, and 20-28, 21-22-5 403 and 22-5 for Awa Island on behalf of K. Sato, N. Oka and M. Yamamoto, respectively, 404 and the Agency for Cultural Affairs; No. 4-413, 4-325 and 4-486.

405

\section{References}

407 Alerstam, T., Hake, M. \& Kjellén, N. (2006). Temporal and spatial patterns of repeated 408 migratory journeys by ospreys. - Anim. Behav. 71: 555-566.

409 Arima, H. \& Sugawa, H. (2004). Correlation between the pitch of calls and external 410 measurements of Streaked Shearwaters Calonectris leucomelas breeding on Kanmuri 411 Island. - Jpn. J. Ornithol. 53: 40-44 (in Japanese with English abstract).

Austin, D., Bowen, W.D. \& McMillan, J.I. (2004). Intraspecific variation in movement patterns: modeling individual behaviour in a large marine predator. - Oikos 105:

417 Battley, P.F. (2006). Consistent annual schedules in a migratory shorebird. - Biol. Lett. 
2: $517-520$.

419 Bauer, S., Ens, B.J. \& Klaassen, M. (2010). Many routes lead to Rome: potential causes 420 for the multi-route migration system of Red Knots, Calidris canutus islandica. $421 \quad$ Ecology 91: 1822-1831.

Bell, A.M., Hankison, S.J. \& Laskowski, K.L. (2009). The repeatability of behaviour: a meta-analysis. - Anim. Behav. 77: 771-783.

Bogdanova, M.I., Daund, F., Newell, M., Phillips, R.A., Harris, M.P. \& Wanless, S. (2011). Seasonal interactions in the black-legged kittiwake, Rissa tridactyla: links between breeding performance and winter distribution. - Proc. R. Soc. B 278: 2412-2418.

Bolnick, D.I., Svanbäck, R., Fordyce, J.A., Yang, L.H., Davis, J.M., Hulsey, C.D. \& Forister, M.L. (2003). The ecology of individuals: incidence and implications of individual specialization. - Am. Nat. 161: 1-28.

Bradshaw, C.J.A., Hindell, M.A., Sumner, M.D. \& Michael, K.J. (2004). Loyalty pays: potential life history consequences of fidelity to marine foraging regions by southern elephant seals. - Anim. Behav. 68: 1349-1360.

Bradshaw, W.E. \& Holzapfel, C.M. (2007). Evolution of animal photoperiodism. Annu. Rev. Ecol. Evol. Syst. 38: 1-25.

Broderick, A.C., Coyne, M.S., Fuller, W.J., Glen, F. \& Godley, B.J. (2007). Fidelity and over-wintering of sea turtles. - Proc. R. Soc. B 274: 1533-1538.

Catry, P., Encarnacão, V., Araújo, A., Fearon, P., Fearon, A., Armelin, M. \& Delaloye, P. (2004). Are long-distance migrant passerines faithful to their stopover sites? - J. 
Avian Biol. 35: 170-181.

441 Catry, P., Dias, M.P., Phillips, R.A. \& Granadeiro, J.P. (2013). Carry-over effects from 442 breeding modulate the annual cycle of a long-distance migrant. An experimental 443 demonstration. - Ecology 94: 1230-1235.

444 Dias, M.P., Granadeiro, J.P., Phillips, R.A., Alonso, H. \& Catry, P. (2011). Breaking the 445 routine: individual Cory's shearwaters shift winter destinations between hemispheres 446 and across ocean basins. - Proc. R. Soc. B 278: 1786-1793.

447 Dingemanse, N.J. \& Réale, D. (2005). Natural selection and animal personality. $448 \quad$ Behaviour 142: 1159-1184.

449 Ghalambor, C.K., McKay, J.K., Carroll, S.P. \& Reznick, D.N. (2007). Adaptive versus 450 non-adaptive phenotypic plasticity and the potential for contemporary adaptation in 451 new environments. Func. Ecol. 21: 394-407.

452 Goymann, W., Helm, B., Jensen, W., Schwabl, I. \& Moore, I.T. (2012). A tropical bird 453 can use the equatorial change in sunrise and suanset times to synchronize its $454 \quad$ circannual clock. - Proc. R. Soc. B 279: 3527-3534.

455 Guilford, T., Freeman, R., Boyle, D., Dean, B., Kirk, H., Phillips, R. \& Perrins, C. 456 (2011). A dispersive migration in the Atlantic puffin and its implications for 457 migratory navigation. - PLoS ONE 6(7): e21336.

458 Gunnarsson, T.G., Gill, J.A., Sigurbjörnsson, T. \& Sutherland, W.J. (2004). Pair bonds: 459 arrival synchrony in migratory birds. - Nature 431: 646.

460 Gwinner, E. (1996). Circadian and circannual programmes in avian migration. - J. Exp. $461 \quad$ Biol. 199: 39-48. 
462 Harrison, X.A., Blount, J.D., Inger, R., Norris, D.R. \& Bearhop, S. (2010). Carry-over 463 effects as drivers of fitness differences in animals. - J. Anim. Ecol. 80: 4-18.

464 Hill, R.D. (1994). Theory of geolocation by light levels. - In: Elephant seals: population 465 ecology, behaviour and physiology (Le Boeuf, B. J. \& Laws R. M., eds). University 466 of California Press, Berkley, p. 227-236.

467 Jorgensen, S.J., Reeb, C.A., Chapple, T.K., Anderson, S., Perle, C., Van Sommeran, 468 S.R., Fritz-Cope, C., Brown, A.C., Klimley, A.P. \& Block, B.A. (2010). Philopatry 469 and migration of Pacific white sharks. - Proc. R. Soc. B 277: 679-688.

470 Kokko, H. (1999). Competition for early arrival in migratory birds. - J. Anim. Ecol. 68: $471940-950$.

472 Le Boeuf, B.J., Crocker, D.E., Costa, D.P., Blackwell, S.B., Webb, P.M. \& Houser, D.S. 473 (2000). Foraging ecology of northern elephant seals. - Ecol. Monogr. 70: 353-382.

474 Longhurst, A.R. \& Pauly, D. (1998). Ecology of Tropical Oceans. - Academic Press, $475 \quad$ London.

476 Møller, A.P. (2001). Heritability of arrival date in a migratory bird. - Proc. R. Soc. $477 \quad$ Lond. B 268: 203-206.

478 Newton, I. (2008). The migration ecology of birds. - Academic Press, London.

479 Oka, N. (2004). The distribution of streaked shearwater (Calonectris leucomelas) 480 colonies, with special attention to population size, area of sea where located and 481 surface water temperature. - J. Yamashina Inst. Ornithol. 35: 164-188. (In Japanese $482 \quad$ with English abstract.)

483 Oka, N., Suginome, H., Jida, N. \& Maruyama, N. (2002). Chick growth and fledgling 
484 performance of streaked shearwaters Calonectris leucomelas on Mikura Island for 485 two breeding seasons. - J. Yamashina Inst. Ornithol. 34: 39-59.

486 Parmesan, C. (2006). Ecological and evolutionary responses to recent climate change. 487 Annu. Rev. Ecol. Evol. Syst. 37: 637-669.

488 Phillips, R.A., Silk, J.R.D., Croxall, J.P., Afanasyev, V. \& Briggs, D.R. (2004). 489 Accuracy of geolocation estimates for flying seabirds. - Mar. Ecol. Prog. Ser. 266: 490 $265-272$.

491 Phillips, R.A., Silk, J.R.D., Croxall, J.P., Afanasyev, V. \& Bennett, V.J. (2005). 492 Summer distribution and migration of nonbreeding albatrosses: individual 493 consistencies and implications for conservation. - Ecology 86: 2386-2396.

494 Phillips, R.A., Bearhop, S., Mcgill, R.A.R. \& Dawson, D.A. (2009). Stable isotopes 495 reveal individual variation in migration strategies and habitat preferences in a suite of 496 seabirds during the nonbreeding period. - Oecologia 160: 795-806.

497 Quillfeldt, P., Voigt, C.C. \& Masello, J.F. (2010). Plasticity versus repeatability in 498 seabird migratory behaviour. - Behav. Ecol. Sociobiol. 64: 1157-1164.

499 Reed, T.E., Waples, R.S., Schindler, D.E., Hard, J.J. \& Kinnison, M.T. (2010). 500 Phenotypic plasticity and population viability: the importance of environmental 501 predictability. - Proc. R. Soc. B 277: 3391-3400.

502 Schofield, G., Hobson, V.J., Fossette, S., Lilley, M.K.S., Katselidis, K.A. \& Hays, G.C. 503 (2010). Fidelity to foraging sites, consistency of migration routes and habitat 504 modulation of home range by sea turtle. - Diversity. Distrib. 16: 840-853.

505 Sherrill-Mix, S.A., James, M.C. \& Myers, R.A. (2008). Migration cues and timing in 
leatherback sea turtles. - Behav. Ecol. 19: 231-236.

507 Stanley, C.Q., MacPherson, M., Fraser, K.C., McKinnon, E.A. \& Stutchbury, B.J.M. 508 (2012). Repeat tracking of individual songbirds reveals consistent migration timing 509 but flexibility in route. - PLoS ONE, 7 (7), e40688.

510 Takahashi, A., Ochi, D., Watanuki, Y., Deguchi, T., Oka, N., Afanasyev, V., Fox, J.W. 511 \& Trathan, P.N. (2008). Post-breeding movement and activities of two streaked 512 shearwaters in the north-western Pacific. - Ornithol. Sci. 7: 29-35.

513 Vardanis, Y., Klaassen, R.H., Strandberg, R. \& Alerstam, T. (2011). Individuality in 514 bird migration: routes and timing. - Biol. Lett. 7: 502-505.

515 Walther, G.R., Post, E., Convey, P., Menzel, A., Parmesan, C., Beebee, T.J.C., 516 Fromentin, J.M., Hoegh-Guldberg, O. \& Bairlein, F. (2002). Ecological responses to 517 recent climate change. - Nature 416: 389-395.

518 Yamamoto, T., Takahashi, A., Katsumata, N., Sato, K. \& Trathan P.N. (2010). At-sea 519 distribution and behavior of streaked shearwaters during the non-breeding period. $520 \quad$ Auk 127: 871-881.

521 Yamamoto, T., Takahashi, A., Oka, N., Iida, T., Katsumata, N., Sato, K. \& Trathan, P.N. 522 (2011). Foraging areas of streaked shearwaters in relation to seasonal changes in the 523 marine environment of the Northwestern Pacific: inter-colony and sex-related 524 differences. - Mar. Ecol. Prog. Ser. 424: 191-204.

525 Yamamoto, T., Takahashi, A., Oka, N., Shirai, M., Yamamoto, M., Katsumata, N., Sato, 526 K., Watanabe, S. \& Trathan, P.N. (2012). Inter-colony differences in the incubation 527 
1

2

3

4

6

7

8

10

11

12

13

14

15

16

17

18

19

20

21

22

23

24

25

26

27

28

29

30

31

32

33

34

35

36

37

38

39

40

41

42

43

44

45

46

47

48

49

50

51

52

53

54

55

56

57

58

59

60

61

62

63

64

65

$528 \quad$ Waterbirds 35: 248-259.

529 Zar, J.H. (1999). Biostatistical analysis, 4th edn. - Prentice Hall, Upper Saddle River, $530 \quad$ NJ.

531 


\section{$532 \quad$ Figure legends}

533 Figure 1. Wintering distribution of streaked shearwaters ( $95 \%$ kernel density maps for 534 each wintering area: the seas off northern New Guinea, Arafura Sea, and South China 535 Sea). Stars indicate the colony location (Sangan, Awa, and Mikura Islands).

537 Figure 2. Example migration tracks for individual streaked shearwaters in successive 538 years. Stars indicate the breeding colony (Sangan, Awa, and Mikura Islands). Red lines 539 represent the migration pathway in the first year, blue lines in the second year, and 540 green line in the third year. Panels (a, c, d, e) show examples of individuals that were 541 faithful to their wintering areas for two successive years, or (b) for three successive 542 years. Birds migrated to (b, e) the seas off northern New Guinea, (a) the South China 543 Sea, and (c, d) the Arafura Sea. In addition, birds from Awa Island exhibited two 544 different migratory routes: (c) a route through the Pacific Ocean, and (d) through the 545 Sea of Japan. Panel (f) shows the migration track of the only bird that changed its 546 wintering destination in the successive year.

548 Figure 3. Repeatability for timings in the migration schedule between two years: (a) 549 departure from the breeding colony, (b) southward migration, (c) northward migration, 550 (d) first return at the breeding colony. White thick and thin circles represent males and 551 females that had chicks in both previous seasons (BB), while black and grey circles 552 represent males and females with no chick in the second previous season (BF), 553 respectively. Statistical results for the repeatability $(r)$ are shown. 
Table 1. Number of geolocator attachment and recovery.

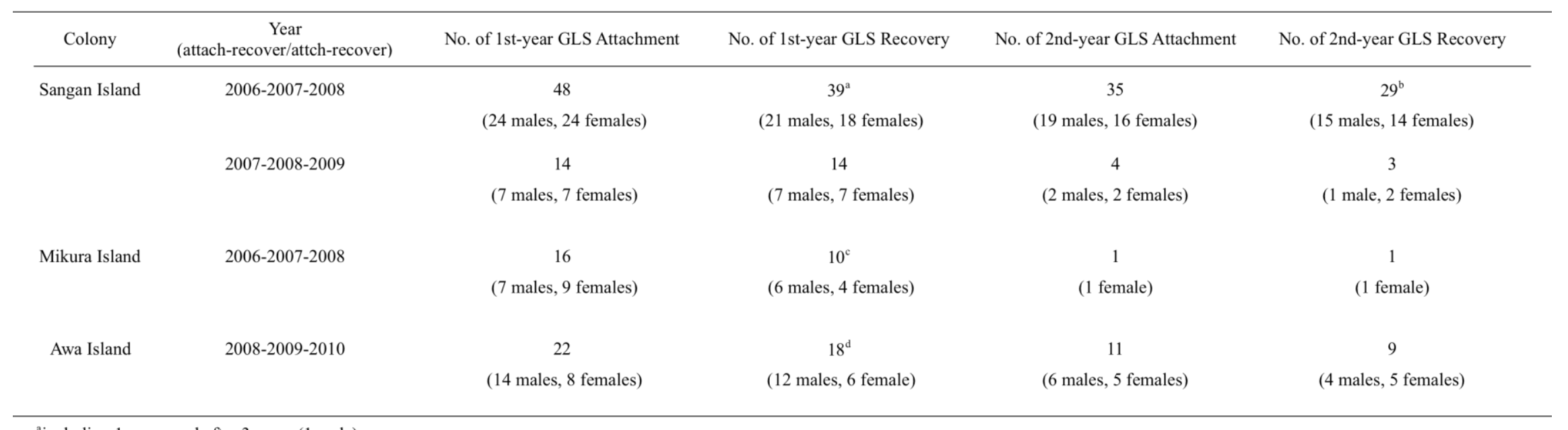

${ }^{a}$ including 1 recovered after 3 years ( 1 male).

including 1 recovered after 2 years $(1 \mathrm{male})$

including 3 recovered after 2 years ( 2 males, 1 female)

dincluding 2 recovered after 2 years ( 2 males). 


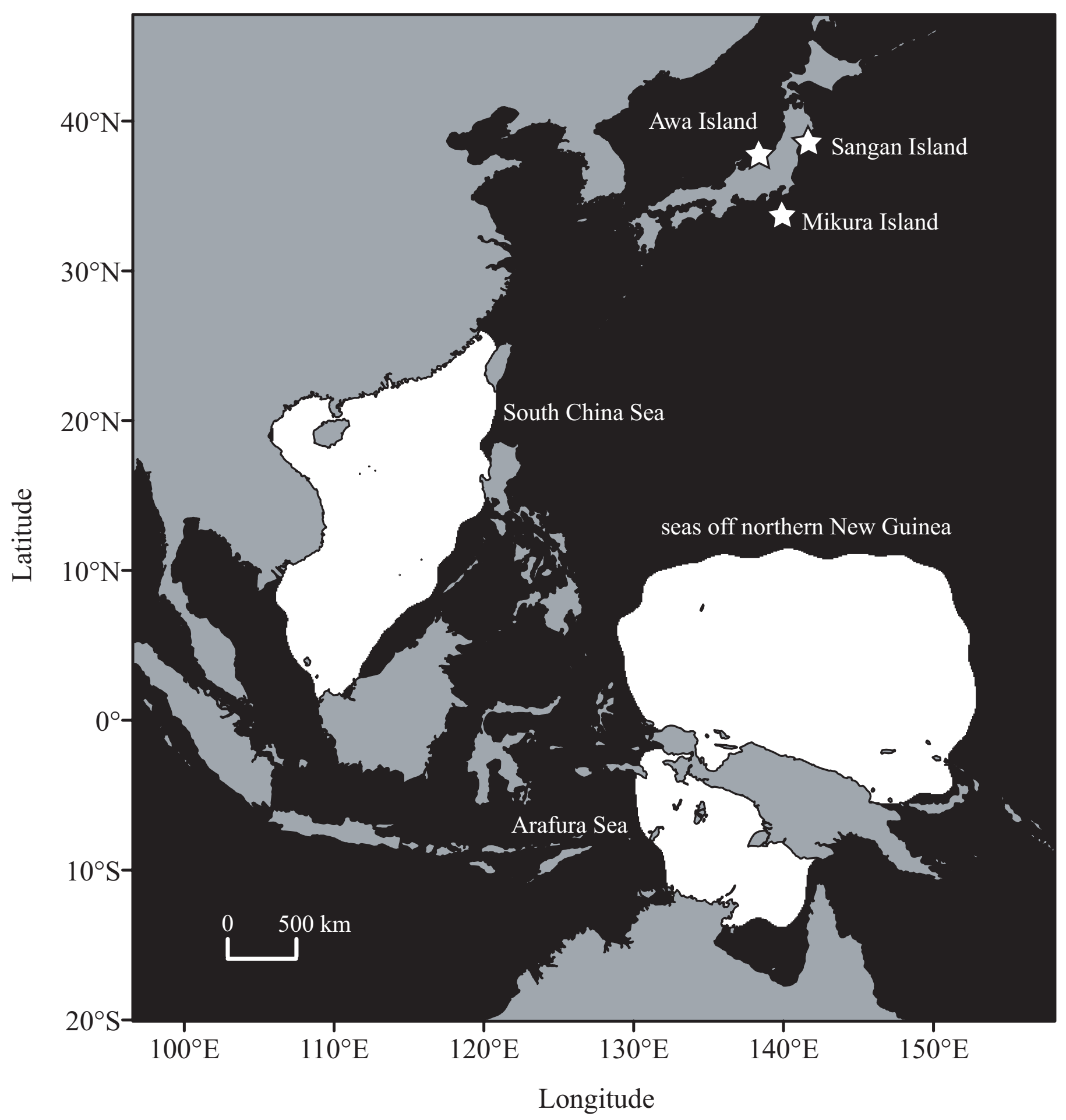

Figure 1 

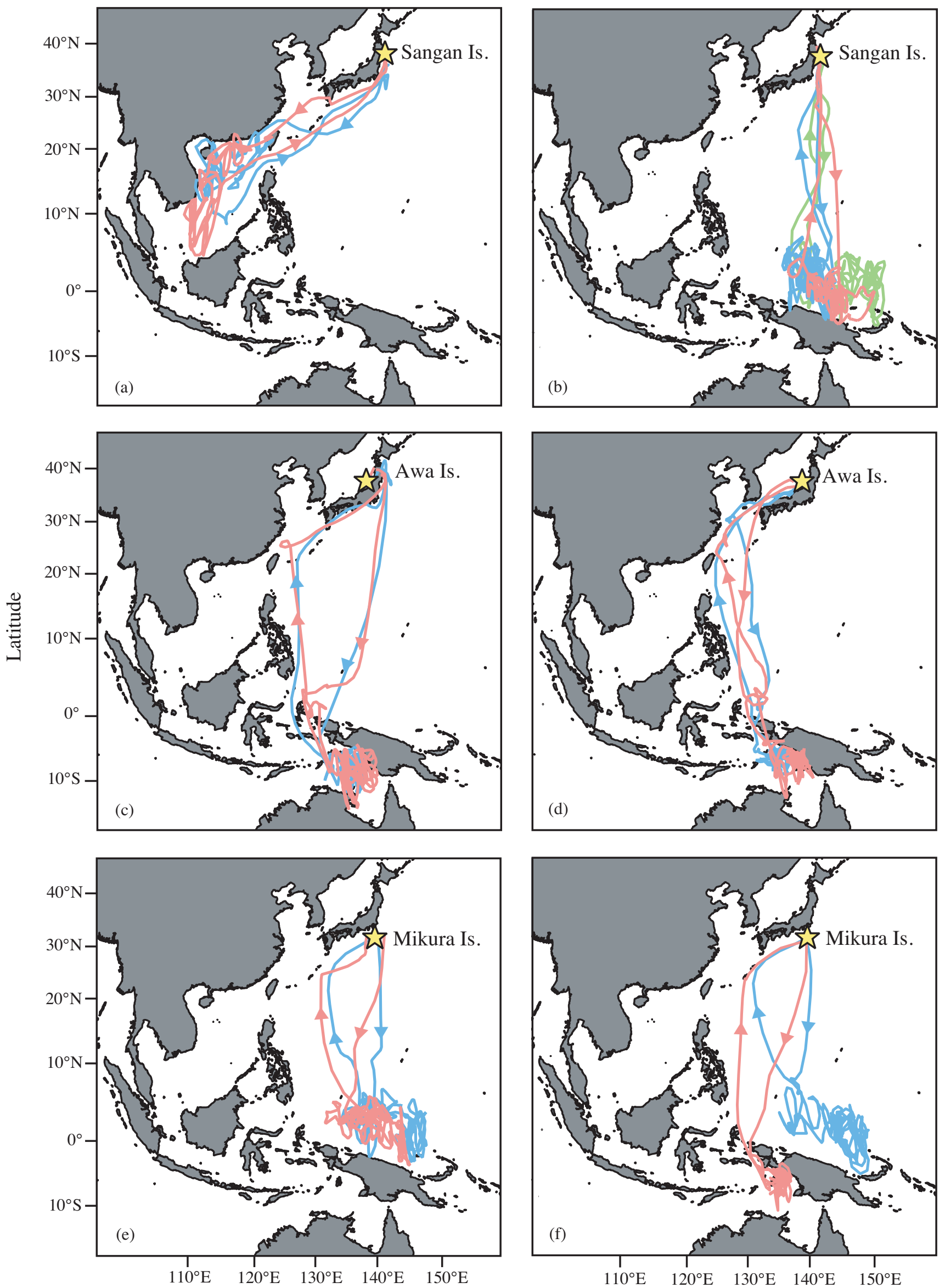

Longitude 


\section{Figure}
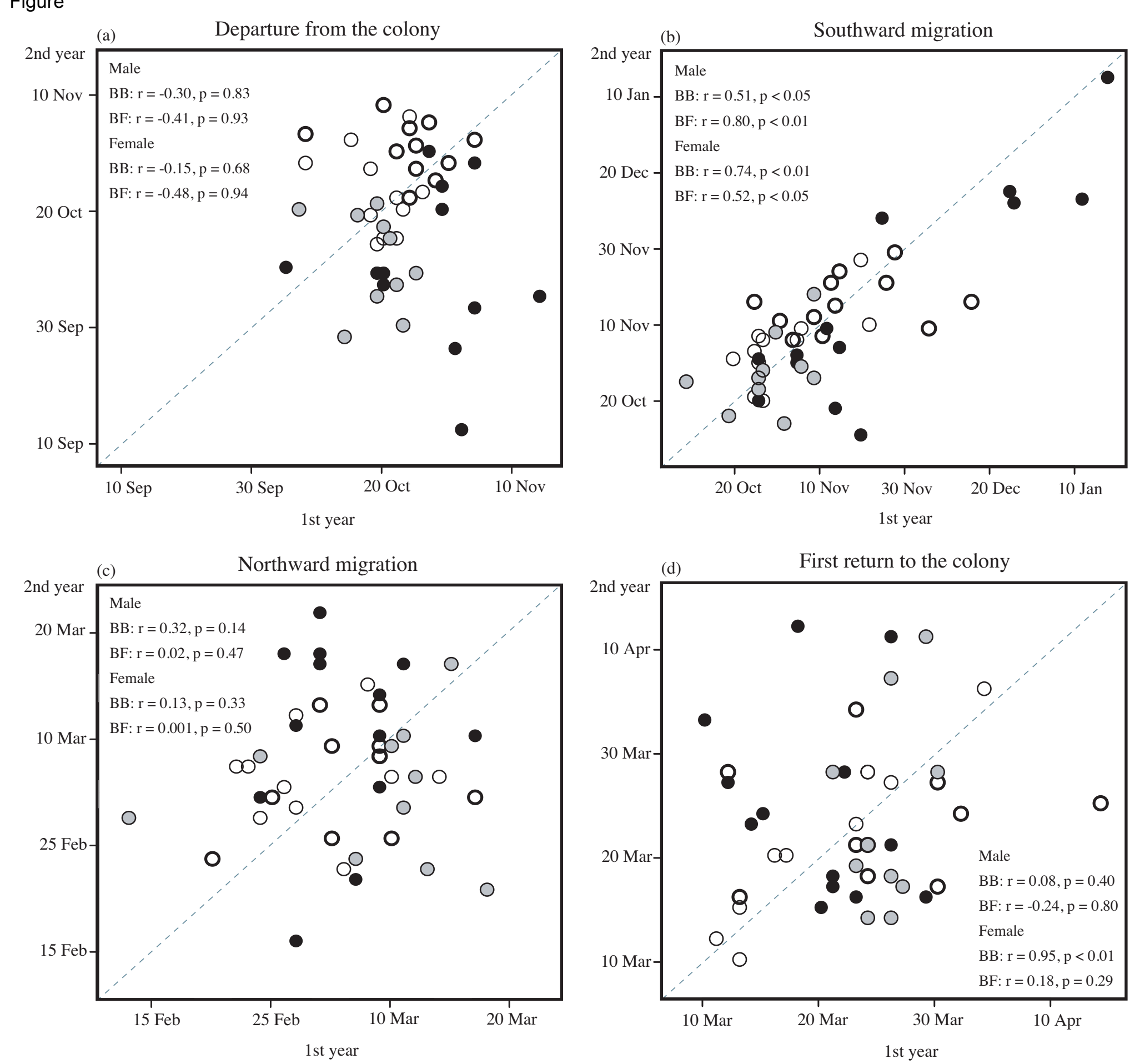

Figure 3 